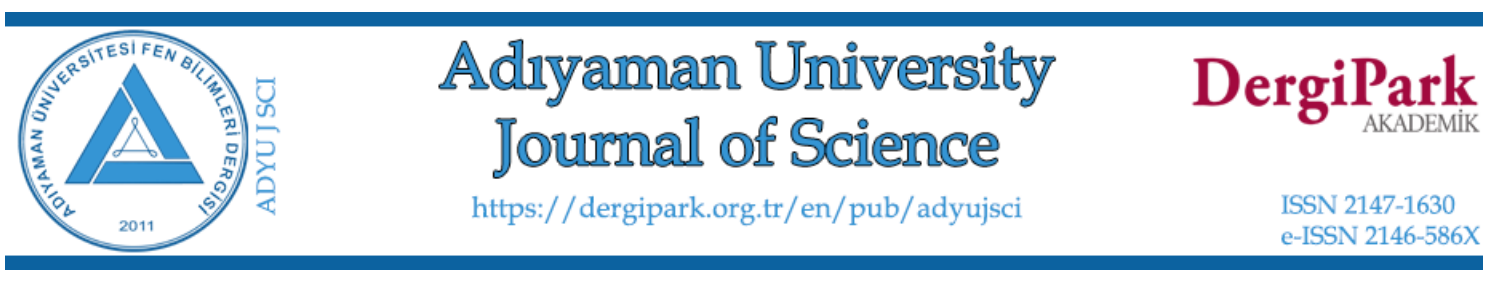

\title{
Some Commutativity Theorems on Lie Ideals of Semiprime Rings
}

\author{
Zeliha BEDİR ${ }^{1, *}$, Öznur GÖLBAŞI ${ }^{2}$ \\ ${ }^{1}$ Cumhuriyet University, Faculty of Science, Department of Mathematics, 58140 Sivas, Turkey \\ zelihabedir@cumhuriyet.edu.tr,ORCID:0000-0002-4346-2331 \\ ${ }^{2}$ Cumhuriyet University, Faculty of Science, Department of Mathematics, 58140 Sivas, Turkey \\ ogolbasi@cumhuriyet.edu.tr,ORCID:0000-0002-9338-6170
}

\begin{tabular}{lll}
\hline Received: 25.10 .2019 & Accepted: 02.12.2020 & Published: 30.12 .2020
\end{tabular}

\begin{abstract}
In this paper, we show that any $U$ noncentral square closed Lie ideal of a 2 -torsion free semiprime ring $R$ contains a nonzero ideal. With this result, some theorems will be extended on the multiplicative generalized derivations of Lie ideals of semiprime rings.

Keywords: Semiprime ring; Lie ideal; Multiplicative generalized derivation.

\section{Yarıasal Halkaların Lie İdealleri Üzerinde Bazı Değişmelilik Teoremleri}

$\ddot{O} \mathbf{z}$

Bu çalışmada, 2 -torsion free bir $R$ yarıasal halkasının kare kapalı merkezi olmayan bir $U$ Lie idealinin, halkanın sıfırdan farklı bir idealini kapsadığı gösterilecektir. Ayrıca bu sonuçla, yarı asal halkaların Lie ideallerinin çarpımsal genelleştirilmiş türevleri üzerine bazı teoremler genelleştirilecektir.
\end{abstract}

Anahtar Kelimeler: Yarıasal halka; Lie ideal; Çarpımsal genelleştirilmiş türev. 


\section{Introduction}

Let $R$ be an assosiative ring with center $Z(R)$. Recall that $R$ is prime if for $a, b \in R, a R b=$ $\{0\}$ implies either $a=0$ and $b=0 . R$ is said to be semiprime if for $a \in R, a R a=\{0\}$ implies $a=0$. Let $R$ be a prime ring. For any pair of elements implies either $x, y \in R$, we shall write $[x, y]$ (resp. $x o y$ ) for the commutator $x y-y x$ (resp., for the Jordan product $x y+y x$ ). An additive subgroup $L$ of $R$ is called a Lie ideal of $R$ if $[u, r] \in L$ for all $u \in L$ and $r \in R$. It is clear that if characteristic of $R$ is 2 , then Lie ideals of $R$ coincide. Lie ideal $U$ of $R$ is said to be square closed if $u^{2} \in U$ for all $u \in U$.

An additive map $d: R \rightarrow R$ is called a derivation if $d(x y)=d(x) y+x d(y)$ holds, for all $x, y \in R$. In [1], Bresar introduced the generalized derivation: An additive mapping $F: R \rightarrow R$ is called a generalized derivation if there exists a derivation (an associated derivation of $F$ ) such that for all $x, y \in R$. The notion a generalized derivation covers both the notions of a derivation and of a left multiplier (i.e., an additive mapping $f: R \rightarrow R$ satisfying $f(x y)=f(x) y$, for all $x, y \in$ $R) . R$ is said to be 2 -torsion-free, if $2 x=0, x \in R$ implies $x=0$.

In the present paper, our main object is to investigate commutativity of semiprime ring satisfying certain differential identities on a nonzero Lie ideal. Let us first recall that the study of commutativity of rings using differential identities goes back to the wellknown Posner's Theorems [2] in which he researched that the presence of a nonzero centralizing derivation $d$ on a prime ring $R$ forces the ring $R$ to be commutative. This result is known by Posner's Second Theorem. Also motivated by this theorem, several authors have introduced new kind of differential identities.

It is important to mention that the study of identities on square closed Lie ideals. Indeed, it is clear that every ideal is a square closed Lie ideal. By [3] Theorem 1.1, every nonzero Jordan ideal of 2-torsion free semiprime rings contains a nonzero ideal. In this paper, we give a similar result for square closed Lie ideals. Then, only the case of ideals could be of interest. Recall the background of study about multiplicative (generalized)-derivations. Firstly, the concept of multiplicative derivation was considered by Daif motivated by Martindale in [4], in the year 1991. Following [5], $d: R \rightarrow R$ is called a multiplicative derivation if $d(x y)=x d(y)+d(x) y$ holds for all pairs $x, y \in R$. These maps are not additive. Later, this mapping were completely described in [6]. Inspired by the definition of multiplicative derivation, Daif and Tammam-El-Sayiad defined the notion of multiplicative generalized derivations. Further, the concept of multiplicative derivations was extended to multiplicative generalized derivations for rings by they in [7] as follows: A mapping $F: R \rightarrow R$ is called a multiplicative generalized derivation if there exists a 
derivation $d$ such that $F(x y)=F(x) y+x d(y)$ holds, for all $x, y \in R$. For completeness of notation, in the present paper, a multiplicative generalized derivation will be denoted by $(F, d)$. A slight generalization of this concept was made by Dhara and Ali [8] by taking $d$ as a mapping ( $d$ isn't necessarily a derivation). Therefore, one may find that the definition of multiplicative generalized derivation covers the notion of multiplicative derivation as well as multiplicative left centralizers. The examples of this concepts can be found in [9]. Obviously, any generalized derivation is a multiplicative generalized derivation, but the converse is not true in general ( see [8]). So, it should be interesting to extend some results concerning derivations and generalized derivations to multiplicative generalized derivations. However, there are only few papers about this subject ( see $[2,6,10-13]$ ), for a partial bibliography). The main objective of the present paper is to explore the cases when a multiplicative generalized derivation $(F, d)$ satisfies the identities:
i) $\left.F\left(\left[u_{1}, u_{2}\right]\right)= \pm u_{1}, d\left(u_{2}\right)\right]$,
ii) $F\left(u_{1} o u_{2}\right)= \pm\left(u_{1} o d\left(u_{2}\right)\right)$,
iii) $F\left(\left[u_{1}, u_{2}\right]\right)= \pm\left(F\left(u_{2}\right) u_{1}\right)$,
iv) $F\left(u_{1} o u_{2}\right)= \pm\left(F\left(u_{2}\right) u_{1}\right)$,

for all $u_{1}, u_{2}$ in some appropriate subsets of $R$. These results investigated by Huang [14]. In this study, these identities are proved without assuming $d(U) \subseteq U$.

In this paper, we will make a lot of calculations with Lie product and Jordan product and mostly use the following identities:

$$
\begin{aligned}
& {\left[u_{1} u_{2}, u_{3}\right]=u_{1}\left[u_{2}, u_{3}\right]+\left[u_{1}, u_{3}\right] u_{2} \text { and }\left[u_{1}, u_{2} u_{3}\right]=u_{2}\left[u_{1}, u_{3}\right]+\left[u_{1}, u_{2}\right] u_{3},} \\
& u_{1} o\left(u_{2} u_{3}\right)=\left(u_{1} o u_{2}\right) u_{3}-u_{2}\left[u_{1}, u_{3}\right]=u_{2}\left(u_{1} o u_{3}\right)+\left[u_{1}, u_{2}\right] u_{3}, \\
& \left(u_{1} u_{2}\right) o u_{3}=u_{1}\left(u_{2} o u_{3}\right)-\left[u_{1}, u_{3}\right] u_{2}=\left(u_{1} o u_{3}\right) u_{2}+u_{1}\left[u_{2}, u_{3}\right] .
\end{aligned}
$$

\section{Results}

The following Lemmas are required for the proof of our main results.

Lemma 1. [7, Lemma 1.3] Let $R$ be a ring with no non-zero nilpotent ideals in which $2 x=$ 0 implies $x=0$. Suppose that $U \neq(0)$ is both a Lie ideal and a subring of $R$. Then either $U \subseteq Z$, the center of $R$, or $U$ contains a non-zero ideal of $R$. 
Lemma 2. [15, Lemma 2.1] Let $R$ be a semiprime ring, $I$ be a nonzero two-sided ideal of $R$ and $a \in R$ such that $a x a=0$, for all $x \in I$, then $a=0$.

Theorem 3. Let $R$ be a 2 -torsion free semiprime ring and $U$ be a noncentral square-closed Lie ideal of $R$. Then $U$ contains a nonzero ideal of $R$.

Proof. By the hypothesis, we have $u^{2} \in U$, for all $u \in U$. Using this, we obtain that

$$
u v+v u=(u+v)^{2}-u^{2}-v^{2} \in U
$$

for all $u, v \in U$. That is,

$$
u v+v u \in U, \text { for all } u, v \in U
$$

On the other hand, using the fact $U$ a Lie ideal of $R$, then

$$
u v-v u \in U, \text { for all } u, v \in U \text {. }
$$

Combining Eqn. (1) and Eqn. (2), we arrive at $2 u v \in U$, for all $u, v \in U$.

Let define $2 U=\{u+u \mid u \in U\}$. We prove that $2 U$ set is subring and Lie ideal of $R$. Indeed, for all $2 u, 2 v \in 2 U$, we have

$$
2 u-2 v=2(u-v) \in 2 U
$$

and

$$
2 u 2 v=(u+u)(v+v)=u v+u v+u v+u v \in 2 U
$$

We conclude that $2 U$ is a subring of $R$.

Moreover, for all $2 u \in 2 U, r \in R$,

$$
[2 u, r]=[u+u, r]=[u, r]+[u, r]=2[u, r] \in 2 U
$$

Therefore, $2 U$ is a Lie ideal of $R$. Furthermore, $2 U \neq(0)$. By Lemma 1 , we conclude that $2 U \subseteq$ $Z$ or $2 U$ contains a nonzero ideal of $R$.

If $2 U \subseteq Z$, then $U \subseteq Z$, which is a contradiction. Hence $2 U$ contains a nonzero ideal of $R$, and so $U$ contains a nonzero ideal of $R$, since $2 U \subseteq U$. 
Theorem 4. Let $R$ be a 2 -torsion free semiprime ring and $U$ be a noncentral square-closed Lie ideal of $R$. If $\mathrm{R}$ admits a multiplicative generalized derivation $(F, d)$ satisfying

$$
F\left(\left[u_{1}, u_{2}\right]\right)= \pm\left[u_{1}, d\left(u_{2}\right)\right], \text { for all } u_{1}, u_{2} \in U
$$

then there exists a nonzero ideal $I$ of $R$ and $d$ is commuting on $I$.

Proof. By Theorem 3, $R$ contains a nonzero ideal $I$ such that $I \subseteq U$. By the hypothesis, we get

$$
F\left(\left[u_{1}, u_{2}\right]\right)=\left[u_{1}, d\left(u_{2}\right)\right], \text { for all } u_{1}, u_{2} \in I \text {. }
$$

Replacing $u_{1}$ by $u_{1}+u_{2}$ in Eqn. (3), we get

$$
F\left(\left[u_{1}, u_{2}\right]+\left[u_{2}, u_{2}\right]\right)=\left[u_{1}, d\left(u_{2}\right)\right]+\left[u_{2}, d\left(u_{2}\right)\right] \text {, for all } u_{1}, u_{2} \in I \text {. }
$$

Using Eqn. (4) in the above relation yields that

$$
\left[u_{2}, d\left(u_{2}\right)\right]=0 \text {, for all } u_{2} \in I \text {. }
$$

It means that $d$ is commuting on $I$.

If we have $F\left(\left[u_{1}, u_{2}\right]\right)+\left[u_{1}, d\left(u_{2}\right)\right]=0$, for all $u_{1}, u_{2} \in U$, applying similar approach above with necessary varitions, we get the required result.

Theorem 5. Let $R$ be a 2 -torsion free semiprime ring and $U$ be a noncentral square-closed Lie ideal of $R$. If $R$ admits a multiplicative generalized derivation $(F, d)$ satisfying

$$
F\left(u_{1} o u_{2}\right)= \pm\left(u_{1} o d\left(u_{2}\right)\right), \text { for all } u_{1}, u_{2} \in U
$$

then there exists a nonzero ideal $I$ of $R$ and $d$ is commuting on $I$.

Proof. By Theorem 3, $R$ contains a nonzero ideal $I$ such that $I \subseteq U$. By the hypothesis, we get

$$
F\left(u_{1} o u_{2}\right)=\left(u_{1} o d\left(u_{2}\right)\right) \text {, for all } u_{1}, u_{2} \in I \text {. }
$$

Taking $u_{1}$ by $u_{1} u_{2}$ in Eqn. (6), we have

$$
\left(u_{1} o u_{2}\right) d\left(u_{2}\right)=u_{1}\left[u_{2}, d\left(u_{2}\right)\right] \text {, for all } u_{1}, u_{2} \in I \text {. }
$$

Replacing $u_{1}$ by $d\left(u_{2}\right) u_{1}$ and using Eqn. (7), we obtain

$$
\left[d\left(u_{2}\right), u_{2}\right] u_{1} d\left(u_{2}\right)=0, \text { for all } u_{1}, u_{2} \in I \text {. }
$$


Right multiplying by $u_{2}$ to the Eqn. (8), we arrive at

$$
\left[d\left(u_{2}\right), u_{2}\right] u_{1} d\left(u_{2}\right) u_{2}=0, \text { for all } u_{1}, u_{2} \in I \text {. }
$$

Taking $u_{1}$ by $u_{1} u_{2}$ in Eqn. (8), we have

$$
\left[d\left(u_{2}\right), u_{2}\right] u_{1} u_{2} d\left(u_{2}\right)=0 \text {, for all } u_{1}, u_{2} \in I \text {. }
$$

Combining Eqn. (9) and Eqn. (10), we obtain

$$
\left[d\left(u_{2}\right), u_{2}\right] I\left[d\left(u_{2}\right), u_{2}\right]=0 \text {, for all } u_{1}, u_{2} \in I \text {. }
$$

Thus, using Lemma 2, we achieved the required result.

Also if we have $F\left(u_{1} o u_{2}\right)+u_{1} o d\left(u_{2}\right)=0$ for all $u_{1}, u_{2} \in U$, then using the same techniques as used above with necessary variations we get the required result.

Theorem 6. Let $R$ be a 2 -torsion free semiprime ring and $U$ be a noncentral square-closed Lie ideal of $R$. If $R$ admits a multiplicative generalized derivation $(F, d)$ satisfying

$$
F\left(\left[u_{1}, u_{2}\right]\right)= \pm\left(F\left(u_{2}\right) u_{1}\right), \text { for all } u_{1}, u_{2} \in U
$$

then there exists a nonzero ideal $I$ of $R$ and $d$ is commuting on $I$.

Proof. By Theorem 3, $R$ contains a nonzero ideal $I$ such that $I \subseteq U$. By the hypothesis, we get

$$
F\left(\left[u_{1}, u_{2}\right]\right)=F\left(u_{2}\right) u_{1} \text {, for all } u_{1}, u_{2} \in I
$$

Replacing $u_{2}$ by $u_{2} u_{1}$ in Eqn. (11), we get

$$
F\left(\left[u_{1}, u_{2}\right]\right) u_{1}+\left[u_{1}, u_{2}\right] d\left(u_{1}\right)=F\left(u_{2}\right) u_{1}^{2}+u_{2} d\left(u_{1}\right) u_{1}, \text { for all } u_{1}, u_{2} \in I \text {. }
$$

Right multiplying by $u_{1}$ to the Eqn. (12), we arrive at

$$
F\left(\left[u_{1}, u_{2}\right]\right) u_{1}=F\left(u_{2}\right) u_{1}^{2} \text {, for all } u_{1}, u_{2} \in I \text {. }
$$

Using last equation in Eqn. (12), we obtain

$$
\left[u_{1}, u_{2}\right] d\left(u_{1}\right)=u_{2} d\left(u_{1}\right) u_{1} \text {, for all } u_{1}, u_{2} \in I \text {. }
$$

Writing $d\left(u_{1}\right) u_{2}$ instead of $u_{2}$ in Eqn. (14) and using Eqn. (14), we have

$$
\left[u_{1}, d\left(u_{1}\right)\right] u_{2} d\left(u_{1}\right)=0 \text {, for all } u_{1}, u_{2} \in I \text {. }
$$


This equation is same as Eqn. (8) in the proof of Theorem 5. Applying the same arguments in the proof of Theorem 5, we get the required result.

On the other hand, it is proved analogously using $F\left(\left[u_{1}, u_{2}\right]\right)+\left(F\left(u_{2}\right) u_{1}\right)=0$, for all $u_{1}, u_{2} \in U$.

By using similar methods in this theorem, the following theorem can be proved.

Theorem 7. Let $R$ be a 2 -torsion free semiprime ring and $U$ be a noncentral square-closed Lie ideal of $R$. If $R$ admits a multiplicative generalized derivation $(F, d)$ satisfying

$$
F\left(\left[u_{1}, u_{2}\right]\right)= \pm\left(F\left(u_{1}\right) u_{2}\right), \text { for all } u_{1}, u_{2} \in U
$$

then there exists a nonzero ideal $I$ of $R$ and $d$ is commuting on $I$.

Theorem 8. Let $R$ be a 2 -torsion free semiprime ring and $U$ be a noncentral square-closed Lie ideal of $R$. If $R$ admits a multiplicative generalized derivation $(F, d)$ satisfying

$$
F\left(u_{1} o u_{2}\right)= \pm\left(F\left(u_{2}\right) u_{1}\right), \text { for all } u_{1}, u_{2} \in U
$$

then there exists a nonzero ideal $I$ of $R$ and $d$ is commuting on $I$.

Proof. By Theorem 3, $R$ contains a nonzero ideal $I$ such that $I \subseteq U$. By the hypothesis, we have

$$
F\left(u_{1} o u_{2}\right)=F\left(u_{2}\right) u_{1} \text {, for all } u_{1}, u_{2} \in I
$$

Taking $u_{2}$ by $u_{2} u_{1}$ in Eqn. (16) and using this, we get

$$
\left(u_{1} o u_{2}\right) d\left(u_{1}\right)=u_{2} d\left(u_{1}\right) u_{1} \text {, for all } u_{1}, u_{2} \in I \text {. }
$$

Writing $d\left(u_{1}\right) u_{2}$ instead of $u_{2}$ in Eqn. (17), we obtain

$$
d\left(u_{1}\right)\left(u_{1} o u_{2}\right) d\left(u_{1}\right)+\left[u_{1}, d\left(u_{1}\right)\right] u_{2} d\left(u_{1}\right)=d\left(u_{1}\right) u_{2} d\left(u_{1}\right) u_{1} \text {, for all } u_{1}, u_{2} \in I \text {. }
$$

Combining Eqn. (17) and Eqn. (18), we arrive at

$$
\left[u_{1}, d\left(u_{1}\right)\right] u_{2} d\left(u_{1}\right)=0, \text { for all } u_{1}, u_{2} \in I \text {. }
$$

This equation is same as Eqn. (8) in the proof of Theorem 5. Applying the same arguments in the proof of Theorem 5, we get the required result. 
Also if we have $F\left(u_{1} o u_{2}\right)+F\left(u_{2}\right) u_{1}=0$, for all $u_{1}, u_{2} \in U$, then in same way, we can prove the same conclusion.

Similary, in view of Theorem 8 , we obtain the following result.

Theorem 9. Let $R$ be a 2 -torsion free semiprime ring and $U$ be a noncentral square-closed Lie ideal of $R$. If $R$ admits a multiplicative generalized derivation $(F, d)$ satisfying

$$
F\left(u_{1} o u_{2}\right)= \pm\left(F\left(u_{1}\right) u_{2}\right), \text { for all } u_{1}, u_{2} \in U
$$

then there exists a nonzero ideal $I$ of $R$ and $d$ is commuting on $I$.

\section{References}

[1] Bresar, M., On the distance of the composition of two derivations to be the generalized derivations, The Glasgow Mathematical Journal, 33, 89-93, 1991.

[2] Posner, E.C., Derivations in Prime Rings, Proceedings of the American Mathematical Society, 8, 1093-1100, 1957.

[3] Herstein, I.N., Topics in ring theory, University of Chicago Press, 1969.

[4] Martindale, W.S., When are multiplicative maps additive, Proceedings of the American Mathematical Society, 21, 695-698, 1969.

[5] Daif, M.N., When is a multiplicative derivation additive, International Journal of Mathematics and Mathematical Sciences, 14(3), 615-618, 1991.

[6] Goldman, H., Semrl, P., Multiplicative derivations on $C(X)$, Monatshefte für Mathematik, 121(3), 189-197, 1969.

[7] Daif, M. N., Tammam El-Sayiad, M.S., Multiplicative generalized derivations which are additive, East-West Journal of Mathematics, 9, 31-37, 1997.

[8] Dhara, B., Ali, S., On multiplicative (generalized)-derivation in prime and semiprime rings, Aequationes mathematicae, 86, 65-79, 2013.

[9] Eremita, D., Ilisevic, D., On additivity of centralizers, Bulletin of the Australian Mathematical Society, 74, 177-184, 2006.

[10] Ali, A., Dhara, B., Ali, F., Multiplicative (generalized)-derivations and left ideals in semiprime rings, Hacettepe Journal of Mathematics and Statistics, 44, 1293-1306, 2015.

[11] Ali, S., Dhara, B., Dar, N.A., Khan, A.N., On Lie ideals with multiplicative (generalized)-derivations in prime and semiprime rings, Beiträge zur Algebra und Geometrie, 56, 325-337, 2015.

[12] Koç, E., Gölbaşı, Ö., Multiplicative generalized derivations on Lie ideals in semiprime rings, Palastine Journal of Mathematics, 6, 219-227, 2017.

[13] Gölbaş1, Ö., Multiplicative generalized derivations on ideals in semiprime rings, Mathematica Slovaca, 66, 1285-1296, 2016.

[14] Huang, S., Derivations on Lie ideals of semiprime rings, Gulf Journal of Mathematics, 6(3), 25-32, 2018. 
Bedir \& Gölbaşı (2020) ADYU J SCI, 10(2), 548-556

[15] Samman, M.S., Alyamani, N., Derivations on semiprime ring, International Journal of Pure and Applied Mathematics, 5(4), 469-477, 2003. 